\title{
Effects of Ultraviolet Exposure Behaviors on Skin Pigmentation and Melanoma
}

\author{
Elena B Hawryluk ${ }^{1}$, Asli Oztan ${ }^{2}$ and David E Fisher ${ }^{3}$
}

${ }^{1}$ Dermatology Program, Boston Children's Hospital, Harvard Medical School, Boston, Massachusetts, USA

${ }^{2}$ F. M. Kirby Neurobiology Center, Department of Neurobiology, Boston Children's Hospital, Harvard Medical School, Boston, Massachusetts, USA

${ }^{3}$ Department of Dermatology, Massachusetts General Hospital, Harvard Medical School, Boston, Massachusetts, USA

*Corresponding author: Elena B Hawryluk, Dermatology Program, Boston Children's Hospital, Harvard Medical School, Boston, Massachusetts, USA, Tel: 617-643-5428; E-mail: Elena.Hawryluk@childrens.harvard.edu

Rec date: May 15, 2014; Acc date: June 20, 2014; Pub date: June 22, 2014

Copyright: (c) 2014 Hawryluk EB, et al. This is an open-access article distributed under the terms of the Creative Commons Attribution License, which permits unrestricted use, distribution, and reproduction in any medium, provided the original author and source are credited.

\begin{abstract}
Tanning refers to the practice of darkening the pigment of one's skin through exposure to natural sunlight or artificial Ultraviolet (UV) radiation. Studies have explored the molecular mechanism of tanning induced by UV radiation exposure. UV radiation triggers DNA damage in the nucleus of keratinocytes, which induces production of melanin by melanocytes. The melanin is then transported to keratinocytes and manifests as darker pigmentation. An increasing amount of data accumulate to support a role for UV exposure in the development of both melanoma and non-melanoma skin cancers. This article reviews the body of literature reporting the effects of UV exposure on melanocyte biology, skin pigmentation, and melanoma.
\end{abstract}

Keywords: Pigmentation; Melanoma; Melanocytes; Indoor Tanning; Ultraviolet; Sunscreen

\section{Abbreviations}

a-MSH: a-Melanocyte-Stimulating Hormone; FDA: Food and Drug Administration; MC1R: Melanocortin 1 Receptor; MITF: Microphthalmia-associated Transcription Factor; POMC: Proopiomelanocortin; SPF: Sun Protection Factor; UPF: Ultraviolet Protection Factor; UV: Ultraviolet

\section{Introduction}

Tanning refers to the practice of darkening one's skin pigmentation through natural sunlight and artificial ultraviolet (UV) exposure. Studies have explored the molecular mechanism of darkening one's skin pigmentation through exposure to UV radiation, and an increasing amount of data supports the role of UV exposure in the development of both melanoma and non-melanoma skin cancers. This article reviews the body of literature reporting the effects of UV exposure on melanocyte biology, skin pigmentation, and melanoma.

Natural sunlight produces UV radiation in the UVA, UVB, and UVC wavelengths. UVA wavelengths (320-400 nm) are effective to induce increased pigmentation and cause indirect DNA damage through the production of free radicals, oxidative damage, and stress as detailed below. UVB radiation (280-320 nm) causes direct DNA damage and mutation, and is associated with sunburns and vitamin D biosynthesis. UVC radiation $(200-280 \mathrm{~nm})$ is largely filtered by the stratospheric ozone, while nearly all UVA and up to $10 \%$ of UVB radiation reaches the Earth's surface. Tanning machines (also called sunlamps or sunbeds), in contrast to topical-based tanning cosmeceuticals, provide exogenous UV radiation with intent to darken skin pigmentation. While topical tanning products do not expose one's skin to increased UV radiation, they can increase susceptibility to the dangerous effects of UV rays, as described in further detail below.

\section{Tanning is a Common Practice}

Tanning is a common practice, and the industry is sizable. The U.S. Food and Drug Administration (FDA) estimates that over 30 million Americans use tanning devices each year, and tanning beds can be found at a variety of venues including tanning salons, beauty salons, spas, and health clubs. The Indoor Tanning Association reports that about $10 \%$ of Americans visit a tanning facility each year, and this large industry includes 19,000 facilities and 160,000 employees [1].

In 2014, Wehner and colleagues performed a systematic review and meta-analysis that included 88 studies and 406,696 participants to determine the prevalence of indoor tanning practices in the United States, Europe, and Australia [2]. These authors found a prevalence of lifetime exposure to indoor tanning of $35.7 \%$ for adults, $55 \%$ for university students, and $19.3 \%$ for adolescents. Indoor tanning exposure over the past year was found to be as high as $14 \%$ for adults, $43.1 \%$ for university students, and $18.3 \%$ for adolescents [2]. A crosssectional interview-based study of adolescents in U.S. cities found that indoor tanning was practiced by $17.1 \%$ of adolescent girls and $3.2 \%$ of adolescent boys in the preceding year. Greater use was found among adolescents who are Caucasian, older, and female; have a greater allowance; have a parent who practices indoor tanning; and live within two miles of an indoor tanning facility [3].

The additional UV exposure experienced by patrons of indoor tanning facilities is significant. Frequent tanners, defined as those with 100 or more sessions total in their lifetime, using modern highpressure sunlamps, were found to have up to 10-times greater annual UV exposure compared to other tanners [4].

\section{Effects of UV Exposure on Melanocytes}

Studies have explored the cellular impact of UV exposure. UVinduced DNA damage activates cellular repair signals that increase skin pigmentation which may function as a barrier against further UV damage. Specifically, UV radiation triggers DNA damage in the nucleus of keratinocytes, which induces production of melanin in 
melanocytes. Melanin is subsequently transported back to the keratinocytes, as summarized in detail below. Human skin has developed several protective mechanisms from UV damage, including DNA repair, apoptosis, cell cycle arrest, and post-translational regulation by microRNAs [5].

Specific UV wavelengths have signature effects on DNA and impart DNA damage by distinct mechanisms, as reviewed in detail elsewhere [6]. Both UVA and UVB radiation are able to generate the formation of cyclobutane pyrimidine dimers to trigger DNA damage. UVA radiation excites endogenous chromophores, which generate reactive oxygen species that cause oxidative DNA damage. UVA-induced damage significantly affects guanine nucleotides resulting in G->T and G->A mutations. UVB targets adjacent pyrimidine nucleotides, causing the formation of cyclobutane pyrimidine dimers and pyrimidine 6-4 pyrimidones, which may either spontaneously revert or trigger DNA repair enzymes. UVB-induced damage generates $\mathrm{C}->\mathrm{T}$ and CC->TT mutations. Solar radiation-induced DNA mutations have been shown to occur with exposure to both fluorescent light and radiation from tanning salon beds [7].

The downstream cellular effects of UV radiation are summarized in Figure 1. DNA damage causes stabilization of the tumor suppressor protein $\mathrm{p} 53$, which activates transcription and increases the expression of the gene encoding proopiomelanocortin (POMC). POMC is cleaved, producing $\alpha$-melanocyte-stimulating hormone $(\alpha-M S H)$ and $\beta$-endorphin (which may relate to the addictive features of tanning). Keratinocytes release $\alpha-\mathrm{MSH}$, which acts on the melanocortin 1 receptor (MC1R) of basal melanocytes. Polymorphic variants of $\mathrm{MC1R}$ produce the red hair/fair skin phenotype, which (because these variants are deficient in signaling response to $\alpha-\mathrm{MSH}$ ) results in an inability to tan. Subsequently, there is an increase of cAMP and transcription of microphthalmia-associated transcription factor (MITF), which promotes transcription of pigmentation genes relevant to the synthesis and transport of melanin. Concurrently, UV radiation causes depolarization of melanocytes via transient receptor protein A1 ion channels, causing increased intracellular calcium, which is required for melanin synthesis [8].

Melanin pigment is packaged into melanosomes and transported out of melanocytes to keratinocytes via dendritic extensions. Recent electron microscopy and cell culture data suggest that melanosomes are transferred via keratinocyte-induced exocytosis, mediated by Rab11b-induced melanosome membrane modeling, with subsequent keratinocyte endocytosis [9]. Within the keratinocyte, pigmentcontaining melanosomes coalesce over the UV-exposed side of the nucleus, manifesting as the skin's tanned appearance and potentially protecting the nucleus from subsequent UV radiation damage. In human subjects, the amount of sun protection provided by facultative pigmentation from repeated UV exposure has been estimated as a sun protection factor (SPF) of 2 among subjects with darker skin types [10]. This mechanism was shown to provide no protection factor from solar simulated radiation-induced DNA damage for individuals with Fitzpatrick phototypes I and II skin [11].

\section{Association of UV Exposure with Melanoma}

The data continue to mount in support of the association of UV exposure with melanoma. The World Health Organization's International Agency for Research and Cancer classified UV radiation as carcinogenic to humans in 2009 [12]. Early studies suggested the association of indoor tanning with cancer in the 1990s. Older tanning lamps (prior to late 1970s) produced a broad spectrum of UV radiation including UVA, UVB, and UVC wavelengths. In an effort to improve tanning effectiveness and safety, newer tanning lamps were modified to minimize UVB rays and emit primarily UVA wavelengths. However, sunbed UVA emission spectra are 10 to 15 times greater than that of the sun [13]. The risk of developing melanoma among users before and after 2000 is similar, which suggests no improvement in safety with the newer technology [14] although it remains possible that differences may appear with longer follow up interval.

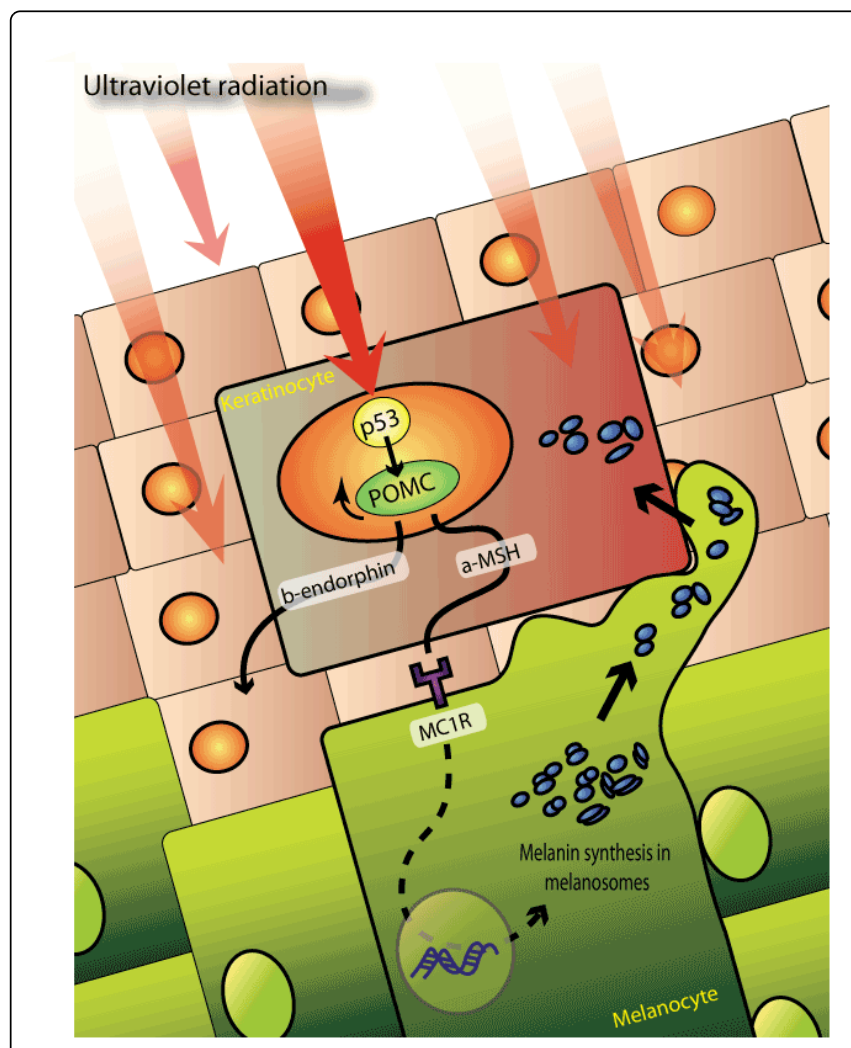

Figure 1: Ultraviolet radiation causes DNA damage that triggers melanin synthesis through the depicted signaling pathway: p53 induces the activation of proopiomelanocortin (POMC), which is cleaved to produce $\alpha$-melanocyte-stimulating hormone ( $\alpha-M S H)$, which activates the melanocyte via the melanocortin 1 receptor (MC1R), causing transcription of genes to synthesize and transport melanin.

The body of epidemiologic literature supporting the relationship of indoor tanning with melanoma was comprehensively reviewed in 2014 by Colantonio and colleagues. Their systematic review and metaanalysis of 31 studies included data on 14,956 melanoma cases and 223,106 controls. These authors found the odds ratio for melanoma associated with ever using an indoor tanning bed was 1.16 [14]. Greater frequency of indoor tanning practice was more strongly associated with melanoma: Subjects who practiced indoor tanning more than 10 times had an increase in the odds ratio to 1.34 [14]. Wehner and colleagues reported the population proportional attributable risk of indoor tanning for melanoma to be between 2.6 and $9.4 \%$, corresponding to over 10,000 melanoma cases each year caused by exposure to indoor tanning among individuals in the United States, Europe, and Australia [2]. 
Recent epidemiology studies have explored the diverse effects of UV exposure in the development of various melanoma subtypes with distinct clinical and mutational profiles, as reviewed by Nikolaou and Stratigos in 2014 [15]. Mounting evidence supports that BRAF mutations are more often detected in melanomas located at body sites with intermittent UV exposure and in patients with intense UV exposure during their youth. Mutations in c-Kit and amplifications of cyclin D1 are more commonly found in melanomas related to chronic exposure to sun, as well as acral and mucosal melanomas.

In addition to the increased risk of melanoma, indoor tanning is associated with a significantly increased risk of squamous cell carcinoma (relative risk 1.67) and basal cell carcinoma (relative risk 1.29 ), according to a meta-analysis of 12 studies with 9,328 cases of non-melanoma skin cancer [16]. This corresponds to over 170,000 cases of non-melanoma skin cancer each year attributed to UV exposure [16].

\section{Approaches to Reduce UV Exposure}

In the United States, the regulation of indoor tanning devices is under state control, and some efforts have been made recently to pass legislation that bans or restricts access to indoor tanning facilities for minors [17]. A cross-sectional study to explore the effectiveness of such bans was performed in 2013, in which a random sample of California tanning facilities (where statewide legislation was passed to ban sunbed use for individuals under age 18 in 2011) were phoned by a study investigator who indicated that she was 17 -years-old. The caller was denied services at $77 \%$ of facilities, suggesting that legislation has made a meaningful impact on access to minors, though the phone conversations revealed inaccurate risk disclosures and claims by the tanning facilities [18]. This study is limited by the nature of phone interviews and does not provide evidence of denial of tanning services when a minor presents to an indoor tanning facility in person. A 2013 telephone-based cross sectional survey of Missouri tanning facilities, where there was no state legislation to restrict tanning access, found that $43 \%$ of salons claimed that there are no risks associated with indoor tanning [19].

Multiple approaches can be utilized to reduce natural UV exposure, in addition to avoiding the exogenous UV exposure of indoor tanning. Various sun-protection measures include use of sunscreens (both chemical and physical UV blockers are available), use of ultraviolet protection factor (UPF)-containing clothing, use of physical barriers such as clothing and cover-ups to block the sun, avoidance of activities during peak UV-exposure hours of the day, and shade-seeking behavior. In a prospective split-nevus study in which nevi were protected partially by sunscreens and physical barriers, both approaches were shown to partially prevent the effects of UVB radiation [20]. Histopathology revealed less melanocytic activation and regression features with protection by physical barrier compared to sunscreen after a single dose of UVB irradiation [20].

Historically, the use of topical sunscreens to lessen melanoma risk has been controversial, with a lack of evidence supporting the intervention; a 2002 meta-analysis including pooled observational studies found no association between sunscreen use and development of melanoma [21]. However, in 2011, authors in Australia provided 10 -year follow up on a cohort study of daily versus discretionary sunscreen application, which demonstrated a significant reduction in invasive melanomas diagnosed at 10 years with daily sunscreen use
[22]. These authors and others found that use of sunscreen is correlated with increased sun exposure $[21,23,24]$.

The general public remains poorly informed regarding sun protection and non-compliant with sun protection measures. Even among patients seeking dermatologic care, a 2014 questionnaire-based study showed deficiency in knowledge and practice. Among 2,215 questionnaire respondents, factors associated with low self-reported adherence to sun protection included age less than 20, age greater than 64, male gender, having lower knowledge about sun protection recommendations and UV-associated risks, and having low UV exposure [24]. Again, the respondents who reported better compliance with sun protection also reported higher sun exposures [24]. Given the association of melanoma with intermittent, high-intensity UV exposures, vacationers are at particular risk. A 2014 cross-sectional survey-based study of departing and returning air passengers and vacationers spending holidays in the tropics and subtropics provided insight into their sun protection behaviors. While almost all vacationers used sunscreen, with fewer using protective clothing and seeking shade, $44 \%$ of returning air passengers reported sunburn during their vacation [25].

Physician counseling provides an opportunity to improve patient knowledge about UV exposure risks. The rate that physicians mention sunscreen during patient visits is quite low, based upon data from a National Ambulatory Medical Care Survey that queried patient outpatient office visits between 1989 and 2010 [26]. Among 18.3 billion patient visits, physicians mentioned sunscreen at $0.07 \%$ of visits in total and at only $0.9 \%$ of visits that were associated with a skin disease diagnosis. Surprisingly, sunscreen counseling was delivered at only $1.6 \%$ of all dermatology visits. Among all visits, counseling was most often delivered to white, elderly patients [26].

Methods to tan the skin in the absence of UV-exposure were reviewed by O'Leary and colleagues in 2014 [27]. Topical cosmeceutical tanning products, which do not provide any UV radiation protection, include water-soluble topical bronzers and longer-acting compounds that produce a tan color when placed on the stratum corneum and fade with the sloughing of dead skin cells. Dihydroxyacetone, a common longer-acting topical tanner, leads to formation of products that can generate free radicals during UV irradiation (180\% increase in additional radicals generated compared to untreated skin) [28], suggesting the need for additional UV protection measures among users of sunless topical tanners. Recently, oral pills containing canthaxanthin or beta-carotene have been used to darken skin color, though side effects can range from retinopathy and aplastic anemia to increased rate of lung cancers in users who smoke. Injectable $\alpha-\mathrm{MSH}$ analogues, such as afamelanotide, are being studied for photoprotective effects for patients with a variety of skin diseases, while unregulated $\alpha$-MSH analogues including melanotan I and II are not approved (unclear safety and efficacy data) [27].

Tanning remains a common practice, despite our understanding of the molecular impact of UV exposure on melanocyte biology and the association with skin cancer and melanoma become increasingly certain with ongoing molecular and epidemiologic data. Ongoing efforts to educate individuals on the dangers of tanning and on approaches to reduce UV exposure through both legislation and sun protection practices should have the potential to mitigate these risks. 


\section{Funding}

EBH is supported by funding from the Dermatology Foundation, and DEF is supported by grants from the NIH. NIH Grant number: 1P01 CA163222 and 2R01 AR043369-17.

\section{References}

1. http://www.theita.com

2. Wehner MR, Chren MM, Nameth D, Choudhry A, Gaskins M, et al. (2014) International prevalence of indoor tanning: a systematic review and meta-analysis. JAMA Dermatol 150: 390-400.

3. Mayer JA, Woodruff SI, Slymen DJ, Sallis JF, Forster JL, et al. (2011) Adolescents' use of indoor tanning: a large-scale evaluation of psychosocial, environmental, and policy-level correlates. Am J Public Health 101: 930-938.

4. Miller SA, Hamilton SL, Wester UG, Cyr WH (1998) An analysis of UVA emissions from sunlamps and the potential importance for melanoma. Photochem Photobiol 68: 63-70.

5. Syed DN, Khan MI, Shabbir M, Mukhtar H (2013) MicroRNAs in skin response to UV radiation. Curr Drug Targets 14: 1128-1134.

6. Garibyan L, Fisher DE (2010) How sunlight causes melanoma. Curr Oncol Rep 12: 319-326.

7. DeMarini DM, Shelton ML, Stankowski LF Jr (1995) Mutation spectra in Salmonella of sunlight, white fluorescent light, and light from tanning salon beds: induction of tandem mutations and role of DNA repair. Mutat Res 327: 131-149.

8. Bellono NW, Oancea E (2013) UV light phototransduction depolarizes human melanocytes. Channels (Austin) 7.

9. Tarafder AK, Bolasco G, Correia MS, Pereira FJ, Iannone L, et al. (2014) Rab11b mediates melanin transfer between donor melanocytes and acceptor keratinocytes via coupled exo/endocytosis. J Invest Dermatol 134: 1056-1066

10. Sheehan JM, Cragg N, Chadwick CA, Potten CS, Young AR (2002) Repeated ultraviolet exposure affords the same protection against DNA photodamage and erythema in human skin types II and IV but is associated with faster DNA repair in skin type IV. J Invest Dermatol 118:825-829.

11. Young AR, Potten CS, Chadwick CA, Murphy GM, Hawk JL, et al. (1991) Photoprotection and 5-MOP photochemoprotection from UVR-induced DNA damage in humans: the role of skin type. J Invest Dermatol 97: 942-948.

12. El Ghissassi F, Baan R, Straif K, Grosse Y, Secretan B, et al. (2009) A review of human carcinogens--part D: radiation. Lancet Oncol 10: 751-752.

13. Gerber B, Mathys P, Moser M, Bressoud D, Braun-Fahrländer C (2002) Ultraviolet emission spectra of sunbeds. Photochem Photobiol 76: 664-668.
14. Colantonio S, Bracken MB, Beecker J (2014) The association of indoor tanning and melanoma in adults: systematic review and meta-analysis. J Am Acad Dermatol 70: 847-857.

15. Nikolaou V, Stratigos AJ (2014) Emerging trends in the epidemiology of melanoma. Br J Dermatol 170: 11-19.

16. Wehner MR, Shive ML, Chren MM, Han J, Qureshi AA, et al. (2012) Indoor tanning and non-melanoma skin cancer: systematic review and meta-analysis. BMJ 345: e5909.

17. Gosis B, Sampson BP2, Seidenberg AB1, Balk SJ3, Gottlieb M4, et al. (2014) Comprehensive evaluation of indoor tanning regulations: a 50state analysis, 2012. J Invest Dermatol 134: 620-627.

18. Grewal SK, Haas AF, Pletcher MJ, Resneck JS Jr (2013) Compliance by California tanning facilities with the nation's first statewide ban on use before the age of 18 years. J Am Acad Dermatol 69: 883-889.

19. Balaraman B, Biesbroeck LK, Lickerman SH, Cornelius LA, Jeffe DB (2013) Practices of unregulated tanning facilities in Missouri: implications for statewide legislation. Pediatrics 131: 415-422.

20. Carrera C, Puig-Butillè JA, Aguilera P, Ogbah Z, Palou J, et al. (2013) Impact of sunscreens on preventing UVR-induced effects in nevi: in vivo study comparing protection using a physical barrier vs sunscreen. JAMA Dermatol 149: 803-813.

21. Huncharek M, Kupelnick B (2002) Use of topical sunscreens and the risk of malignant melanoma: a meta-analysis of 9067 patients from 11 casecontrol studies. Am J Public Health 92: 1173-1177.

22. Green AC, Williams GM, Logan V, Strutton GM (2011) Reduced melanoma after regular sunscreen use: randomized trial follow-up. J Clin Oncol 29: 257-263.

23. Autier P, Doré JF, Reis AC, Grivegnée A, Ollivaud L, et al. (2000) Sunscreen use and intentional exposure to ultraviolet $\mathrm{A}$ and $\mathrm{B}$ radiation: a double blind randomized trial using personal dosimeters. Br J Cancer 83: $1243-1248$

24. Sattler U, Thellier S, Sibaud V, Taieb C, Mery S, et al. (2014) Factors associated with sun protection compliance: results from a nationwide cross-sectional evaluation of 2215 patients from a dermatological consultation. Br J Dermatol.

25. Reinau D, Achermann C, Arnet N, Meier CR, Hatz C, et al. (2014) Sun protective behaviour of vacationers spending holidays in the tropics and subtropics. Br J Dermatol.

26. Akamine KL, Gustafson CJ1, Davis SA1, Levender MM1, Feldman SR2 (2014) Trends in sunscreen recommendation among US physicians. JAMA Dermatol 150: 51-55.

27. O'Leary RE, Diehl J, Levins PC2 (2014) Update on tanning: More risks, fewer benefits. J Am Acad Dermatol 70: 562-568.

28. Jung K, Seifert M, Herrling T, Fuchs J (2008) UV-generated free radicals (FR) in skin: their prevention by sunscreens and their induction by selftanning agents. Spectrochim Acta A Mol Biomol Spectrosc 69: 1423-1428. 